\title{
Evolving DSM Diagnostic Criteria for PTSD: Relevance for Assessment and Treatment
}

Michelle J. Bovin, $P h D^{1,3, *}$

Brian P. Marx, PhD $D^{1,3}$

Paula P. Schnurr, PhD ${ }^{2,4}$

\author{
Address \\ ${ }^{*}, 1$ National Center for PTSD, VA Boston Healthcare System, Boston, Massachusetts \\ Email: Michelle.Bovin@va.gov \\ ${ }^{2}$ National Center for PTSD, VA Medical Center, White River Junction, Vermont, USA \\ ${ }^{3}$ Boston University School of Medicine, Boston, Massachusetts \\ ${ }^{4}$ Geisel School of Medicine at Dartmouth, Hanover, NH, USA
}

Published online: 3 February 2015

(C) Springer International Publishing AG (outside the USA) 2015

This article is part of the Topical Collection on Post-Traumatic Stress Disorders

Keywords PTSD · Assessment · DSM-5 - Treatment

\section{Opinion statement}

Careful assessment of PTSD is crucial before, during, and after treatment. In doing so, the use of a multi-method assessment approach that incorporates (semi)structured clinical interviews, self-report instruments, and even psychophysiological assessment is ideal. The changes to the PTSD diagnosis as introduced in the recently revised Diagnostic and Statistical Manual of Mental Disorders have important implications for PTSD assessment. We encourage the clinicians to be mindful of these changes and rely on the new norms and cut-off scores when available, rather than relying on the old heuristics for determining a PTSD diagnosis. By being aware of the changes to the diagnosis and taking these into consideration when choosing a treatment and when evaluating the effect of the treatment, the practitioners will be able to continue to administer the PTSD treatment successfully.

\section{Introduction}

Posttraumatic stress disorder (PTSD) is a debilitating condition, which if left untreated, can persist for many years [1-3]. Therefore, the field has focused its attention on developing a range of treatments that can successfully reduce PTSD symptoms. Of these, the cognitive behavioral therapies have received the most empirical support [4], particularly prolonged exposure (PE) [5], cognitive processing therapy (CPT) [6], and Stress Inoculation Training (SIT) $[7,8]$. Pharmacotherapy has also been used as an intervention for PTSD. Although 
generally not as effective as cognitive-behavioral treatments [9], empirical evidence does support the use of both selective serotonin reuptake inhibitors (SSRIs) and serotonin-norepinephrine reuptake inhibitors (SNRIs) as first-line treatments for PTSD. Further, adjunctive treatment with atypical antipsychotics for patients who are unresponsive to SSRIs and SNRIs has shown some promise.

Although researchers have devoted considerable attention to developing treatments for PTSD, less attention has been given to the role of assessment in the successful treatment of PTSD. This oversight is noteworthy in that careful assessment is crucial to identifying an appropriate treatment, monitoring progress throughout the treatment, and determining whether additional interventions are warranted after the completion of treatment. In this review, we briefly describe how to construct an appropriate PTSD assessment battery and the importance of using this battery or components of this battery throughout treatment. In addition, we discuss how the recent introduction of the fifth edition of the Diagnostic and Statistical Manual of Mental Disorders (DSM-5) [10] has affected assessment in the context of treatment.

\section{Assessment in PTSD treatment}

Assessment of PTSD can be conducted using a range of available instruments, each possessing varying strengths and weaknesses. Structured, standardized diagnostic interviews are considered the "gold standard" for assessing PTSD symptoms. However, because such structured interviews are time-consuming and must be administered by a trained clinician, it may not be feasible to administer them in every situation. Self-report measures of PTSD symptomatology can be used when time and resources are scarcer but they have their own limitations. Specifically, self-report instruments have fixed item content and rating scale formats, and their accuracy is contingent upon the patient understanding each item and answering truthfully.

Assessment in the context of treatment is not limited to the measurement of symptoms of PTSD and psychiatric comorbidity. For example, clinicians' use the Subjective Units of Discomfort scale (SUDs) [11] to determine patients' level of distress during imaginal and in vivo exposure exercises. Although research has shown that using the SUDs in this fashion is an appropriate clinical practice [12], clinicians should not assume that an individual's self-report of distress can completely substitute for objective measurement of physiological arousal to trauma-related stimuli. As such, clinicians should strive to employ multiple methods of assessment to adequately capture data from the three response systems (self-reported emotional experience, expressive behavior, and objective physiological indicators of distress or arousal during the treatment of those with PTSD) [13, 14]. An important caveat of collecting psychophysiological data, however, is that collecting such data requires extensive training and expensive equipment. Further, because it cannot be used to reliably discriminate those with PTSD from those without PTSD, psychophysiological assessment may not be informative at the individual level.

In line with a multimethod assessment approach, Weathers et al. [15•] provided nine suggestions for creating an appropriate PTSD assessment battery that takes the setting, population, and application into account. These recommendations include (1) establish explicit goals (i.e., determine the purpose of the assessment); (2) consider the target population and assessment context, including age, gender, trauma type, and assessment setting; (3) consider the available resources, including the availability of trained personnel and the time 
available for the assessment; (4) enhance compliance with the assessment (i.e., ensure the patient is invested in the assessment); (5) use an interview whenever possible; (6) use a DSM-correspondent self-report measure whenever possible (although instruments which assess PTSD but are not DSM-correspondent are available, it is best to use a DSM-correspondent instrument because they directly assess all PTSD symptoms); (7) use the most appropriate scoring rule for a given application (i.e., it is essential to ensure that the scoring rule being used for each measure reflects the population being assessed); (8) use multiple measures whenever possible (this is a key point, because the use of a multimeasure approach eliminates the bias associated with any given instrument); and (9) evaluate response bias (e.g., it is important to assess for malingering, particularly among patients who may be seeking disability compensation). These guidelines will help the assessor to design an appropriate multimethod PTSD assessment battery.

The next issue an assessor must consider is the frequency with which the patient is assessed. In a treatment setting, continuous assessment of PTSD symptoms (i.e., assessment before, during, and after treatment) is crucial because it provides important information to both patient and practitioner about how the chosen intervention is working. Prior to treatment, a thorough and accurate assessment of PTSD and other psychiatric comorbidity is essential for choosing appropriate treatment targets. Although a patient may appear to have PTSD as a primary diagnosis, a comprehensive assessment with attention to differential diagnosis may reveal that, although the patient may experience a number of PTSD-like symptoms, those symptoms are better explained by an alternative diagnosis (e.g., depression) for which the optimal treatment may differ. Further, a comprehensive baseline assessment may reveal important information about the overall severity, course, and chronicity of symptoms (as well as which symptoms are particularly debilitating) that may inform the treatment approach. The baseline assessment also may determine that other clinically relevant issues (suicidal ideation) or comorbidities (e.g., chronic substance use) should be addressed prior to the beginning of treatment for PTSD or concurrently with PTSD treatment. Assessment prior to treatment allows the provider insight into the patient's baseline level of functioning in interpersonal, occupational, and other important domains.

Assessment during the course of PTSD treatment is necessary for several reasons. First, assessment during the treatment provides a litmus test for the progress the patient is making in the therapy; that is, the practitioner can use frequent and ongoing assessments to determine if the patient's symptoms are decreasing as a function of treatment. Assessment during treatment can also help the clinician gauge patients' level engagement. For example, patients completing a course of PE often report an exacerbation of symptoms after their first imaginal exposure session [16]. There may be several reasons why a particular patient may not demonstrate this pattern of symptom increases. One possible reason may be that the patient is not fully participating in the treatment, either because it is too painful (e.g., effortful avoidance) or because he/she cannot engage (e.g., dissociation). If the patient is, in fact, not engaging, this is important information for the practitioner. This information can only be gleaned by frequent assessment during treatment. Although no specific guidelines exist for how often assessments should be conducted during treatment, many PTSD psychotherapy protocols recommend brief self-report assessments 
at the end of each therapy session [17]. Other exposure-based treatment protocols require the use of frequent situational (e.g., SUDs) ratings during imaginal and in vivo exposure exercises [16]. A more comprehensive PTSD assessment, including a structured interview, midway through treatment, is frequently undertaken [16], although it may not always be feasible. Frequent assessment during treatment is also recommended if the intervention is pharmaceutical; initial reductions in symptoms may be the result of a placebo effect, whereas lack of symptom reduction over time may indicate that another intervention (either psychotherapy or pharmacotherapy) may be more effective.

After treatment, assessment of the symptoms that were targeted during treatment allows the clinician to determine the degree of symptom improvement by calculating reliable change and clinically significant change scores. Reliable change scores indicate the extent to which changes on scores of a particular measure are greater than the expected measurement error (i.e., change from pre-treatment to post-treatment exceeds 1.96 times the standard error of the difference) [18]. Clinically, significant change scores indicate the extent to which the patient's end state scores on a particular measure compare with scores observed in clinically meaningful comparison groups (e.g., the patient's pretreatment to post-treatment scores are separated by at least two standard deviations from the original mean; the patient is within two standard deviations of a normative mean sample post-treatment; and the patient has a greater likelihood of being in the normative distribution than a clinical distribution post-treatment) $[19,20]$.

Whereas reliable change provides information about whether the treatment decreased the patient's symptoms, clinically significant change provides information about whether the symptom decrease improved the patient's level of functioning. Both are important post-treatment indicators of change attributed to the treatment. Post-treatment assessments can also provide information about symptoms or conditions that may require additional interventions. Determining what to assess post-treatment is contingent upon the assessment question. For example, if the only outcome of interest is PTSD, the posttreatment assessment would focus on assessing the symptoms of this disorder. However, if other conditions or symptoms are also treatment targets, then a broader assessment battery would be warranted. Similarly, the number of follow-up assessments may be tied to the circumstances under which the assessments are being conducted. Ideally, patients would participate in a comprehensive assessment immediately following treatment, as well as at several follow-up periods (e.g., 3-month post-treatment, 6-month post-treatment, and 1 -year post-treatment). This provides information about how the patient is functioning immediately after treatment, and whether the gains from treatment are maintained over time. However, other factors, including available personnel and patient willingness, may limit the number of follow-up assessments that can be administered.

The treatment context may influence the assessment battery used at each assessment period. In general, it is important to use the instruments which are well validated for the target population to ensure that interpretations of results are appropriate. Resources also may, in part, determine what instruments are used and how frequently they can be administered. For example, the availability of trained clinicians who can administer structured clinical interviews will determine how often these gold standard instruments can be utilized. 


\section{Changes to the PTSD diagnosis and implications for assessment in PTSD treatment}

In 2013, the American Psychiatric Association introduced the fifth edition of the Diagnostic and Statistical Manual for Mental Disorders (DSM-5) [10]. In this version, there are some notable changes to the PTSD diagnosis, including that it is no longer included as an anxiety disorder. Instead, it is included in a new category called the "traumaand stressor-related disorders" along with acute stress disorder and the adjustment disorders.

In terms of the actual diagnostic criteria, the first notable change concerns the stressor criterion (Criterion A). In DSM-IV, Criterion A was divided into two parts: an objective component (Criterion A1) and a subjective component (Criterion A2). Criterion A1 required that "the person experienced, witnessed, or was confronted with an event or events that involved actual or threatened death or serious injury, or a threat to the physical integrity of self or others" [21]. Criterion A2 further required that "the person's response involved intense fear, helplessness or horror" [21]. Criterion A in DSM-5 now requires:

exposure to actual or threatened death, serious injury, or sexual violence in one (or more) of the following ways: 1 . directly experiencing the traumatic event(s); 2. witnessing, in person, the events(s) as it occurred to others; 3. learning that the traumatic event(s) occurred to a close family member or close friend. In cases of actual or threatened death of a family member of friend, the event(s) must have been violent or accidental; 4. experiencing repeated or extreme exposure to aversive details of the traumatic event(s) (e.g., first responders collecting human remains; police officers repeatedly exposed to details of child abuse). [10]

Criterion A2 has been removed completely from DSM-5 because it did not improve the PTSD diagnostic accuracy [22•].

Other diagnostic criteria were also changed. Criterion B, previously referred to as "re-experiencing symptoms" in DSM-IV, was renamed "intrusion symptoms" to emphasize the ruminative qualities of the symptoms included in this cluster [23•]. In the DSM-IV, the two avoidance symptoms were included as part of the seven symptoms which captured both avoidance of stimuli associated with the trauma as well as numbing of general responsiveness (DSM-IV; Criterion C). In the DSM-5, these two effortful avoidance symptoms are the only once included in Criterion C, which was retitled as "Persistent avoidance of stimuli associated with the traumatic event."

The other symptoms that were once included in Criterion $\mathrm{C}$ have now been moved to a new criterion (Criterion D), called "Negative alterations in cognitions and mood." Three of these symptoms have not been altered: dissociative amnesia (DSM-5 symptom D1); diminished interest or participation in significant activities (DSM-5 symptom D5); and feelings of detachment or estrangement from others (DSM-5 symptom D6). One symptom, restricted range of affect, was altered slightly to assess a persistent inability to experience positive emotions (DSM-5 symptom D7), and another, sense of foreshortened future, was expanded in scope and significantly revised to capture persistent and 
exaggerated negative beliefs about oneself, other people, or the world (DSM-5 symptom D2) [23•]. In addition, two new items were added to this criterion: inappropriate self- or other-blame (DSM-5 symptom D3) and persistent negative emotional state (DSM-5 symptom D4).

The hyperarousal criterion (Criterion D in DSM-IV) is now Criterion E, "marked alterations in arousal and reactivity associated with the traumatic event." This criterion has two notable changes. The first is the modification of the DSM-IV anger item from a more emotion-focused symptom, "irritability or outbursts of anger" [21], to a more behaviorally-focused symptom, "irritable behavior and angry outbursts (with little or no provocation) typically expressed as verbal or physical aggression toward people or objects" [10]. The second is the addition of a new symptom (E2), reckless or self-destructive behavior.

The DSM-5 diagnostic algorithm for PTSD was also affected by these changes. In DSM-IV, a patient qualified as meeting criteria for PTSD if he/she had a Criterion A event and at least one Criterion B symptom, at least three Criterion $\mathrm{C}$ symptoms, and at least two Criterion D symptoms that continued for at least 1 month post-trauma and caused clinically significant distress or impairment. In DSM-5, the algorithm for PTSD now requires that in order to meet the criteria for the diagnosis, in addition to experiencing a Criterion A event, an individual has at least one Criterion B symptom, at least one Criterion C symptom, at least two Criterion D symptoms, and at least two Criterion E symptoms, which have persisted at for at least 1 month after the trauma and which cause clinically significant distress or impairment. The major change is the requirement of at least one avoidance symptom.

One final change to the PTSD diagnosis has important implications for treatment and therefore assessment in the context of treatment. In DSM-5, the PTSD diagnosis now includes a "with dissociative symptoms" specifier. Individuals who meet the criteria for this specifier also experience persistent dissociative symptoms of detachment from their bodies and/or the world around them [10]. This subtype was added to DSM-5 as a result of studies suggesting that there may be two distinct types of PTSD, those with a "hyperarousal" PTSD response and those with a "dissociative" PTSD response, and that these two types demonstrate quantifiable differences in central nervous system activation $[22 \bullet, 24 \bullet, 25 \bullet]$.

The changes made to the PTSD diagnosis have important implications for PTSD assessment. In the following section, we describe the manner in which some of these changes to the diagnosis have particular implications for PTSD assessment within the context of treatment (see Table 1 for a summary of these changes and their implications for assessment).

\section{The stressor criterion}

Because of the aforementioned changes to Criterion A, the events were qualify as a traumatic stress or are now much clearer and more conservative. That is, each category of exposure is defined, specifications are detailed regarding what events will qualify for the potentially broadest categories (e.g., learned about), and examples are provided for the new category (i.e., experiencing exposure to aversive details). Further, the removal of Criterion A2 has placed the focus of the trauma on 
Table 1. Summary of recommendations to clinicians regarding DSM-5 changes and implications for assessment

\section{DSM-5 Changes to the PTSD diagnosis}

Stressor criterion

- Events that qualify as traumatic are clearer and more conservative

- Focus on objective event (removal of Criterion A2)

Criterion D: Negative Alterations in Cognitions and Mood

- Gauges persistent and exaggerated negative beliefs (D2)

- Captures inappropriate blame (D3)

- Assesses persistent negative emotional state (D4)

Changes to the Arousal Criterion: Symptoms E1 and E2

- Focus on behavioral expressions of anger (E1)

- Assessment of reckless or self-destructive behavior (E2)

Introduction of a Dissociative Subtype

- Individuals experience detachment from their bodies and/or

the world around them

Changes to the DSM PTSD Algorithm

- To meet the criteria for PTSD, patient must have:

${ }^{\circ}$ A Criterion A event

-At least 1 Criterion B symptom

- At least 1 Criterion $C$ symptom

- At least 2 Criterion D symptoms

- At least 2 Criterion E symptoms

- Symptoms that persisted for at least 1 month post-trauma

and which cause clinically significant distress/impairment
Implications of assessment in PTSD treatment

- Aids in choosing a traumatic event for treatment focus

- Assists in determining if symptoms remaining post-treatment are tied to a trauma or the result of a different disorder

- Identification of cognitive distortions which are the focus of many PTSD treatments

- Recognition that PTSD may not be fear-based; may influence therapeutic intervention

- Provides information regarding potential therapy-interfering behaviors

- Can inform treatment choice

- Provides information as to whether patients are engaging in non-effortful avoidance

- Assessment instruments are being updated

- New norms and cut-off scores being developed

the objective event, rather than the individual's subjective experience of the event.

These changes to the stressor criterion have important assessment implications. Prior to treatment, it will help to ensure that an event that is indeed traumatic is chosen as the focus (a requirement for most PTSD psychotherapy protocols). Further, at post-treatment, the clarification of Criterion A will assist the practitioners in determining whether remaining symptoms are best conceptualized as PTSD (i.e., they are tied to a traumatic event) or if they are the result of a different disorder (e.g., depression) which requires a different intervention.

\section{Criterion D: Negative alterations in cognitions and mood}

Many PTSD psychotherapy protocols $[6,16]$ focus on changing both assimilated beliefs (i.e., inappropriate blame) and overgeneralized beliefs (dysfunctional beliefs which resulted from the trauma) that are now characterized by PTSD Criteria D2 and D3. Therefore, an accurate assessment of PTSD Criteria D2 and D3 prior to initiating treatment will provide insight into the specific beliefs that should be targeted throughout the course of treatment. Of course, these symptoms should be regularly assessed during therapy to determine the extent to which they are being modified by the intervention. Finally, because dysfunctional cognitions which remain after treatment may continue to 
produce high levels of distress [4], clinicians should assess for the continued existence of these beliefs during follow-up assessments.

The addition of symptom D4, the presence of a "persistent negative emotional state (e.g., fear, horror, anger, guilt, or shame)" [10], also has important implications for PTSD assessment within the context of treatment. The introduction of D4 is a reflection of the re-categorization of PTSD as a "trauma- and stressorrelated disorder" rather than as an anxiety disorder. This new classification is a reflection of research that has suggested that, although the reaction to a traumatic event may be anxiety- or fear-based, it may also be most prominently expressed through dysphoric, externalizing, or dissociative symptom profiles [10].

Symptom D4 captures a broader range of emotions beyond fear and is therefore relevant to a larger group of individuals with PTSD. In the initial assessment, this examination of a range of strong emotions will therefore give the clinician a better sense of how the event has affected the patient. It is also possible that D4 will assist the practitioners in choosing an appropriate intervention. For example, exposure interventions may be more appropriate for fearbased presentations, whereas cognitive-focused interventions may be more appropriate for presentations associated with other emotions (e.g., guilt). However, this possibility awaits empirical testing. During treatment, assessment of this symptom will also give the provider a more nuanced conceptualization of how the patient is responding to the therapeutic intervention; it can provide insight into the patient's full emotional experience. Finally, post-treatment, assessment of a range of strong negative emotions can provide information as to what has been resolved as a result of the intervention (e.g., guilt) and what remains an ongoing clinical consideration (e.g., anger).

\section{Changes to the arousal criterion: symptoms E1 and E2}

Symptoms E1 and E2 are particularly relevant in the context of treatment, because they can speak to potential therapy-interfering behaviors. Behaviors such as yelling at, or threatening, the therapist (which would qualify as symptom E1) or engaging in parasuicidal behaviors or substance abuse (which would qualify as symptom E2) can each interfere with progress in therapy [26, 27]. The knowledge of these behaviors is essential for the provider, because it may be necessary to treat these behaviors either prior to or concurrent with PTSD treatment. Careful assessment of these symptoms, ideally using a structured interview, can provide insight into the existence of these behaviors and allow the practitioner to develop a treatment plan accordingly. Assessment of these behaviors is also relevant both during and after treatment. During treatment, a decrease of these symptoms may be an indication that the patient is responding to PTSD protocol. Post-treatment, in addition to providing a measure of treatment progress, assessment of these behaviors, can provide insight into whether supplementary treatment protocols (e.g., anger management) may be warranted.

\section{Introduction of a dissociative subtype}

Initial research has suggested that individuals with this subtype may respond differently to PTSD interventions than those without this subtype. Specifically, 
in a randomized controlled trial (RCT) in which 150 women with PTSD due to a sexual or physical assault were randomized to CPT, cognitive therapy only (CPT-C), and written accounts only, researchers found that women who endorsed low pre-treatment levels of dissociation responded most efficiently to CPT-C, whereas women with the highest levels of dissociation responded better to CPT [28]. Other studies have demonstrated that dissociative symptoms can affect the success of PTSD treatments. Cloitre and her colleagues [29] conducted an RCT comparing three interventions: Skills Training in Affective and Interpersonal Regulation (STAIR) followed by Narrative Story Telling (NST; STAIR/ NST), STAIR followed by supportive counseling, and supportive counseling followed by NST. The authors found that at post-treatment, the participants with high dissociation at baseline in the STAIR/NST condition continued to improve at follow-up, those treated with STAIR and then supportive counseling maintained their gains and those treated with supportive counseling and then NST demonstrated a loss of post-treatment PTSD symptom gains. Although this research is preliminary, it does suggest that the knowledge of whether a patient has the dissociative subtype of PTSD should be taken into consideration when choosing an appropriate treatment.

Knowledge regarding the presence of the dissociative subtype can only be gleaned through careful assessment. Although most self-report measures do not assess for dissociative symptoms, many of the structured interviews for PTSD do. Assessing for this subtype is particularly important during the initial assessment, so that the appropriate treatment protocol can be chosen. Continued assessment for dissociative symptoms is also relevant throughout the therapy. Dissociation has been conceptualized as a non-effortful avoidance strategy which may reduce awareness of aversive emotions [22 $\bullet$,30]. Therefore, assessment of dissociative symptoms throughout the treatment may provide insight into whether the patient is continuing to avoid trauma-related material through dissociation. Assessment of dissociative symptoms is also relevant at the end of therapy, as a further measure of the success of the protocol. Indeed, research suggests that successful PTSD treatment can often reduce symptoms of dissociation $[28,31,32]$.

\section{Other considerations: DSM-5 PTSD assessment instruments}

In this review, we have discussed how specific content changes to the diagnostic structure of PTSD may impact assessment within the context of treatment. However, it is also worth noting that with the revisions to the PTSD diagnosis come with the advent of new assessment tools designed to assess PTSD symptoms and identify those who meet the criteria for the diagnosis and changes to these tools will also affect assessment within the context of treatment. DSM-5 versions of several assessment instruments, including the Clinician Administered PTSD Scale (CAPS-5) [33], the Structured Clinical Interview for DSM Disorders (SCID-5) [34], the PTSD Checklist (PCL-5) [35], and the Posttraumatic Symptom Scale - Interview Version (PSS-I-5) [36], have already been developed, and psychometric testing of these measures has begun.

Although many of these measures will retain their basic format, the changes to the PTSD diagnosis will affect the interpretation of these instruments. In particular, the severity scores and cut-off scores which separate individuals with 
and without the PTSD will change as a product of altering the diagnosis. This is a key consideration when evaluating whether the patient is appropriate for PTSD treatment, whether the patient is responding to treatment, and after treatment is concluded, determining whether the patient requires additional interventions. The practitioners can ensure that they use the updated measures appropriately by referring to published norms and cut-off scores for the new instruments, rather than assuming that the new instruments will have the same psychometric properties as those that assessed the DSM-IV diagnosis of PTSD.

Prior to the introduction of standardized norms and cut-off scores, the practitioners can still use the DSM-5 measures effectively. To do so, the practitioners are encouraged to consider how patient profiles match with the new DSM-5 PTSD algorithm, rather than using the old cut-off scores as a heuristic. Further, improvement during and after therapy can be ascertained by comparing the individual's pre-treatment scores with those during and after therapy. Additionally, the DSM-5 algorithm can be used to determine whether the patient continues to meet criteria for PTSD after therapy.

\section{Summary and conclusions}

Our review has highlighted the importance of assessment at all stages of PTSD treatment. Prior to treatment, assessment is essential for determining the appropriate intervention. During treatment, assessment can provide essential information about a patient's progress as well as his/ her engagement in the intervention. Finally, post-treatment assessment can be used to provide evidence of reliable and clinically significant change. Assessment at this stage can also be useful for determining whether additional interventions are warranted.

The introduction of DSM-5 has brought several significant changes to the PTSD diagnosis. This review has highlighted four changes which have particular relevance for PTSD assessment within the context of treatment: changes to the stressor criterion, the introduction of both the negative alterations in cognitions and mood criterion (Criterion D), the changes made to the arousal criterion (Criterion E), and the dissociative subtype. The assessment of each of these additions can assist practitioners in providing more effective treatment to patients meeting criteria for PTSD.

Our review also highlights the changes that are being made to the instruments designed to quantify the PTSD diagnosis and PTSD symptom severity. With the introduction of new instruments reflective of DSM-5 criteria come changes to well-known cut-off scores. We recommend caution in using these new instruments, and encourage the clinicians to rely on new norms and cut-off scores when available, rather than relying on the old heuristics. Prior to the introduction of this new psychometric data, we encourage the practitioners to evaluate the assessment data in light of the DSM-5 algorithm and on a case-bycase basis.

Overall, it is essential to rely on careful and comprehensive assessments to ensure that treatment is conducted as effectively as possible. The changes to the PTSD diagnosis as detailed in DSM-5 introduce new considerations when conceptualizing PTSD. By being aware of the changes to the diagnosis and taking these into consideration when choosing a treatment and when 
evaluating the effect of the treatment, the practitioners will be able to continue to administer PTSD treatment successfully.

\section{Compliance with Ethics Guidelines}

\section{Conflict of Interest}

Michelle J. Bovin declares that she has no conflict of interest.

Brian P. Marx declares that he has no conflict of interest.

Paula P. Schnurr declares that she has no conflict of interest.

\section{Human and Animal Rights and Informed Consent}

This article does not contain any studies with animal subjects performed by any of the authors. With regard to the authors' research cited in this paper, all procedures were followed in accordance with the ethical standards of the responsible committee on human experimentation and with the Helsinki Declaration of 1975, as revised in 2000 and 2008.

\section{References and Recommended Reading}

Papers of particular interest, published recently, have been highlighted as:

- Of importance

1. Dirkzwager AJE, Bramsen I, Van Der Ploeg HM. The longitudinal course of posttraumatic stress disorder symptoms among aging military veterans. J Nerv Ment Dis. 2001;189:846-53. doi:10.1097/00005053200112000-00006.

2. Port CL, Engdahl B, Frazier PA. A longitudinal and retrospective study of PTSD among older prisoners of war. Am J Psychiatry. 2001;158:1474-9. doi:10.1176/ appi.ajp.158.9.1474.

3. Solomon Z, Mikulincer M. Trajectories of PTSD: a 20year longitudinal study. Am J Psychiatry. 2006;163:659-66.

4. Cahill SP, Rothbaum BO, Resick PA, Follette VM. Cognitive-behavioral therapy for adults. In: Foa EB, Keane TM, Friedman MJ, Cohen JA eds. Effective Treatments For PTSD: Practice Guidelines From The International Society For Traumatic Stress Studies (2nd Ed.). New York, NY: Guilford; 2009:139-222. Available from: http://www.amazon.com. Accessed September 8, 2014.

5. Foa EB, Hembree EA, Rothbaum, BO, eds. Prolonged Exposure Therapy For PTSD: Emotional Processing Of Traumatic Experiences: Therapist Guide. New York, NY: Oxford University Press; 2007. Available from: http:// www.amazon.com. Accessed September 8, 2014.

6. Resick PA, Schnicke MK. Cognitive processing therapy for sexual assault victims. J Consult Clin Psych. 1992;60:748-756. http://dx.doi.org.ezproxy.bu.edu/ 10.1037/0022-006X.60.5.748.

7. Kilpatrick DG, Veronen LJ, Resick PA. Psychological sequelae to rape: Assessment and treatment strategies.
In: Doleys DM, Meredith RL, Ciminero AR eds. Behavioral Medicine: Assessment And Treatment Strategies. New York, NY: Plenum Press; 1982:473-497. Available from: http://www.amazon.com. Accessed September 8, 2014.

8. Meichenbaum D. Cognitive Behavior Modification. Morristown, NJ: General Learning Press; 1974 . Available from: http://www.amazon.com. Accessed September 8, 2014.

9. Foa EB, Keane TM, Friedman MJ, Cohen JA eds. Effective Treatments For PTSD: Practice Guidelines From The International Society For Traumatic Stress Studies (2nd Ed.). New York, NY: Guilford; 2009:139-222. Available from: http://www.amazon.com. Accessed September 8, 2014.

10. American Psychiatric Association. Diagnostic And Statistical Manual Of Mental Disorders: DSM-5 (5th ed.). Arlington, VA: American Psychiatric Publishing, Inc.; 2013. Available from: http://www.amazon.com. Accessed September 8, 2014.

This is the most recent publication of the Diagnostic and Statistical Manual of Mental Disorders - the DSM-5. The new PTSD diagnostic requirements are presented here.

11. Wolpe J. Psychotherapy By Reciprocal Inhibition. Stanford, CA: Stanford Univer. Press; 1958. doi:10. 1037/10575-013 Available from: http://www.amazon. com. Accessed September 8, 2014.

12. Marx BP, Bovin MJ, Keane TM, et al. Concordance between physiological arousal and subjective distress among Vietnam combat veterans undergoing challenge testing for PTSD. J Traum Stress. 2012;25:41625. doi:10.1002/jts.21729. 
13. Keane TM, Wolfe J, Taylor KL. Post-traumatic stress disorder: Evidence for diagnostic validity and methods of psychological assessment. J Clin Psychol. 1987;43:32-43. http://dx.doi.org.ezproxy.bu.edu/10. 1002/1097-4679(198701)43:1\&lt;32::AIDJCLP2270430106\&gt;3.0.CO;2-X.

14. Sloan DM, Kring, AM. Measuring changes in emotion during psychotherapy: Conceptual and methodological issues. Clin Psychol-Sci Pr. 2007;14:307-322. http://dx.doi.org.ezproxy.bu.edu/10.1111/j.14682850.2007.00092.x.

15. Weathers FW, Keane TM, Foa EB. Assessment and diagnosis of adults. In: Foa EB, Keane TM, Friedman MJ, Cohen JA eds. Effective Treatments For PTSD: Practice Guidelines From The International Society For Traumatic Stress Studies (2nd Ed.). New York, NY: Guilford; 2009:23-61. Available from: http://www.amazon.com. Accessed September 8, 2014.

This chapter provides an overview of the conceptual and practical considerations involved in designing and implementing an assessment protocol for trauma and PTSD.

16. Foa EB, Rothbaum BO. Treating The Trauma Of Rape: Cognitive-Behavioral Therapy For PTSD. New York, NY: Guilford; 1998. Available from: http://www. amazon.com. Accessed September 8, 2014.

17. Resick PA, Monson CM, Chard KM. Cognitive Processing Therapy Veteran/Military Version: Therapist's Manual. Washington, DC: Department of Veterans' Affairs; 2008. Available from: http://www.psych. ryerson.ca/cptcanadastudy/CPT_Canada_Study/ Study_Materials_files/Basic\%20Therapist $\%$ 20Manual\%20Text_title\%20page\%20updated.pdf. Accessed September 8, 2014.

18. Jacobson NS, Truax P. Clinical significance: A statistical approach to defining meaningful change in psychotherapy research. J Consult Clin Psych. 1991;59:12-19. http://dx.doi.org.ezproxy.bu.edu/10.1037/0022-006X. 59.1.12.

19. Evans C, Margison F, Barkham M. The contribution of reliable and clinically significant change methods to evidence-based mental health. Evid Based Mental Health. 1998;1:70-2. doi:10.1136/ebmh.1.3.70.

20. Jacobson NS, Follette WC, Revenstorf D. Psychotherapy outcome research: Methods for reporting variability and evaluating clinical significance. Behav Ther. 1984;15:336-352. http://dx.doi.org.ezproxy.bu.edu/ 10.1016/S0005-7894(84)80002-7.

21. American Psychiatric Association. Diagnostic And Statistical Manual Of Mental Disorders: DSM-IV (4th ed.). Washington, DC: American Psychiatric Publishing, Inc; 1994. Available from: http://www.amazon.com. Accessed September 8, 2014.

22.• Friedman MJ, Resick PA, Bryant RA, Brewin, CR. Considering PTSD for DSM-5. Depress Anxiety. 2011; 128:750-769. http://dx.doi.org.ezproxy.bu.edu/10. 1002/da.20767

This article reviews empirical literature concerning the DSM-
IV-TR diagnostic criteria for PTSD, and describes how this literature influenced the changes to the diagnosis in DSM-5.

23. Miller MW, Wolf EJ, Friedman MJ, et al. The prevalence and latent structure of proposed DSM-5 posttraumatic stress disorder symptoms in U.S. national and veteran samples. Psychol Trauma. 2013;5:501-512. http:// dx.doi.org.ezproxy.bu.edu/10.1037/a0029730.

This article describes two internet-based surveys that were conducted to provide preliminary information about how the changes to the PTSD diagnosis in DSM-5 might impact PTSD prevalence and to clarify the latent structure of the new symptom set. This article does an excellent job of detailing the changes to the PTSD diagnosis that were presented in DSM-5. 24. Wolf EJ, Lunney CA, Miller MW, Resick PA, Friedman MJ, Schnurr PP. The dissociative subtype of PTSD: A replication and extension. Depress Anxiety. 2012;29:679-688. http://dx.doi.org.ezproxy.bu.edu/ 10.1002/da.21946.

This article presents an evaluation of the evidence for a dissociative subtype of PTSD as well as describing the association of the dissociative subtype with personality disorder comorbidity. It provides a review of the empirical support which led to the addition of a dissociative subtype of PTSD in DSM-5.

25. Wolf EJ, Miller MW, Reardon AF, Ryabchenko KA, Castillo D, Freund R. A latent class analysis of dissociation and posttraumatic stress disorder: Evidence for a dissociative subtype. JAMA-J Am Med Assoc. 2012;69:698-705.

26. Linehan MM. Cognitive-Behavioral Treatment Of Borderline Personality Disorder. New York, NY: Guilford; 1993. Available from: http://www.amazon.com. Accessed September 8, 2014.

27. Foa EB, Riggs DS, Massie ED, Yarczower M. The impact of fear activation and anger on the efficacy of exposure treatment for posttraumatic stress disorder. Behav Ther. 1995;26:487-499. http://dx.doi.org.ezproxy.bu.edu/ 10.1016/S0005-7894(05)80096-6.

28. Resick PA, Suvak MK, Johnides BD, Mitchell KS, Iverson KM. The impact of dissociation on PTSD treatment with cognitive processing therapy. Depress Anxiety. 2012;29:718-730. http://

dx.doi.org.ezproxy.bu.edu/10.1002/da.21938.

29. Cloitre M, Petkova E, Wang J, Lu F. An examination of the influence of a sequential treatment on the course and impact of dissociation among women with PTSD related to childhood abuse. Depress Anxiety. 2012;29:709-717. http://dx.doi.org.ezproxy.bu.edu/ 10.1002/da.21920.

30. Griffin MG, Resick PA, Mechanic MB. Objective assessment of peritraumatic dissociation: Psychophysiological indicators. Am J Psychiatry. 1997;154:1081-8.

31. Chard KM. An evaluation of cognitive processing therapy for the treatment of posttraumatic stress disorder related to childhood sexual abuse. J Consult Clin Psych. 2005;73:965-71. doi:10.1037/0022-006X.73.5. 965.

32. Cloitre M, Koenen KC, Cohen LR, Han, H. Skills training in affective and interpersonal regulation followed 
by exposure: A phase-based treatment for PTSD related to childhood abuse. J Consult Clin Psych.

2002;70:1067-1074. http://

dx.doi.org.ezproxy.bu.edu/10.1037/0022-006X.70.5. 1067.

33. Weathers FW, Blake DD, Schnurr PP, Kaloupek DG, Marx BP, Keane TM. Clinician-Administered PTSD Scale for DSM-5: Past month version. Unpublished manuscript; 2013.
34. First MB, Williams JBW, Karg RS, Spitzer RL. Structured Clinical Interview for DSM-5 Disorders (SCID-5), Research Version. Unpublished manuscript; 2014.

35. Weathers FW, Litz BT, Keane TM, Palmieri PA, Marx BP, Schnurr PP. The PTSD Checklist for DSM-5. Unpublished manuscript; 2013.

36. Foa EB, McLean C. Reliability and validity of the PTSD Symptom Scale-Interview Version for DSM-5. Unpublished manuscript; 2014. 